\title{
Optimal investment with derivative securities
}

\author{
Aytaç İlhan $^{1}$, Mattias Jonsson ${ }^{2}$, Ronnie Sircar ${ }^{3}$ \\ 1 Mathematical Institute, University of Oxford, Oxford, OX1 3LB, UK \\ (e- mail: ilhan@maths.ox.ac.uk) \\ 2 Department of Mathematics, University of Michigan, Ann Arbor, MI 48109-1109, USA \\ (e-mail: mattiasj@umich.edu) \\ 3 Department of Operations Research \& Financial Engineering, Princeton University, E-Quad, \\ Princeton, NJ 08544, USA (e-mail: sircar@ princeton.edu)
}

\begin{abstract}
We consider an investor who maximizes expected exponential utility of terminal wealth, combining a static position in derivative securities with a traditional dynamic trading strategy in stocks. Our main result, obtained by studying the strict concavity of the utility-indifference price as a function of the static positions, is that, in a quite general incomplete arbitrage-free market, there exists a unique optimal strategy for the investor.
\end{abstract}

Key words: Utility maximization, convex duality, incomplete markets, indifference price

JEL Classification: G11,G13

Mathematics Subject Classification (2000): 91B16, 91B28, 49N15

\section{Introduction}

Consider an investor who tries to maximize expected utility from wealth at a certain time in the future from a self-financing trading strategy. If the instruments available to the investor are a finite number of risky assets (stocks) and one risk-free asset (the money market account), then the problem of finding the optimal strategy is classical and has been extensively studied: we refer to [19] for a recent survey.

In many cases, however, the investor may also be able to invest in derivative securities. As an example, it is common for traders to buy options positions like butterfly spreads (a particular combination of three call or three put options) to indirectly trade volatility. Such investment opportunities are of particular interest

First and third author partially supported by NSF grant DMS-0306357. Second author partially supported by NSF grant DMS-0200614. 
in incomplete markets, in which case the payoff cannot, in general, be replicated by a trading strategy in the underlyings.

Our concern in this paper is to find optimal trading strategies in the underlying assets as well as finitely many derivative securities. In practice, derivatives trades typically incur relatively large transaction costs, so while we allow for dynamic trading in the stocks, whose lower transaction costs are neglected at first pass, we restrict our attention to static positions in the derivative securities.

We work with exponential utility, $U(x)=-e^{-\gamma x}$, with $\gamma>0$ the risk aversion parameter. Let

$$
X_{t}^{\theta, x}=x+\int_{0}^{t} \theta \cdot d S, \quad 0 \leq t \leq T
$$

be the wealth process from a self-financing trading strategy $\theta$ (see Definition 4.1 for the set of permitted strategies), starting with initial wealth $x$. We shall denote by $G_{1}, \cdots, G_{n}$ the bounded payoffs of the derivative securities. We write $G=$ $\left(G_{1}, \cdots, G_{n}\right)$.

The investor is then trying to maximize, for fixed $x$, the quantity

$$
E\left[U\left(X_{T}^{\theta, x-\lambda \cdot p}+\lambda \cdot G\right)\right]
$$

over dynamic trading strategies $\theta$ and static derivatives positions $\lambda=\left(\lambda_{1}, \ldots, \lambda_{n}\right)$. Here $p=\left(p_{1}, \ldots, p_{n}\right)$ is the market price vector of $G$ and "." denotes the usual inner product on $\mathbf{R}^{n}$. We have assumed the market prices derivatives linearly, which is certainly true for moderate quantities we would expect to be demanded by a small investor in the absence of arbitrage opportunities.

Our main result is that, given a no-arbitrage price vector $p$ for $G$, there exists a unique derivatives position $\lambda^{*}=\lambda^{*}(p)$ together with a dynamic trading strategy $\theta^{\lambda^{*}}$, maximizing the expected utility above. See Theorem 3.1 for a precise statement.

As in much previous work on optimal investment strategies, we make essential use of convex duality in the proof of the main result; in particular we exploit the relation between exponential utility and relative entropy as studied in e.g. [3,6, $8,13,18]$. It turns out that, in order to find the optimal static derivatives position, we need to study the (buyer's) indifference price $b(\lambda)$, the price for which our investor is indifferent, in the sense of maximum expected utility, to investing in the derivatives position $\lambda \cdot G$.

Indifference pricing was introduced by Hodges and Neuberger [9] and has received considerable attention recently. It is closely related to marginal utilitybased pricing, sometimes known as Davis fair pricing, after it was proposed in [5]. In the case of exponential utility, key properties were established by Rouge and El Karoui [18] and Becherer [3], formulas for some specific models are given in [15] and some bounds and asymptotic approximations for stochastic volatility models appear in [22]. Hugonnier et al. [11] consider marginal pricing under general utility functions on $\mathbf{R}_{+}$and establish conditions for uniqueness. Barrieu and El Karoui $[1,2]$ study the problem of optimal design of derivative contracts between two 
parties with different utility functions, and extend to the case of general convex risk measures.

For our problem of optimal investment in derivatives, we need more precise information than we were able to locate in the literature. Specifically, we show that $\lambda \mapsto b(\lambda)$ is strictly concave and admits a gradient $\nabla b(\lambda)$ at every point. Related results on strict concavity for utility functions defined on $\mathbf{R}_{+}$are given by Hugonnier and Kramkov [10]. The gradient $\nabla b(\lambda)$ has a natural interpretation as the Davis fair price, but for a buyer with a different ( $\lambda$-dependent) subjective probability measure: see Theorem 5.1 for details. The optimal derivatives position $\lambda^{*}(p)$ is then the solution to $\nabla b\left(\lambda^{*}\right)=p$. In this paper, we present the mathematical details. For practical considerations and computations within Markovian stochastic volatility models, as well as models with non-traded assets, we refer to our companion paper [12].

\section{The market}

We consider a finite horizon $T<\infty$ and a market consisting of $d+1$ tradeable instruments: a money-market account and $d$ stocks, modeled by a $d$-dimensional locally bounded semimartingale $S$ on a filtered probability space $(\Omega, \mathcal{F}, \mathbb{F}, P)$, where the filtration $\mathbb{F}=\left(\mathcal{F}_{t}\right)_{0 \leq t \leq T}$ satisfies the usual conditions. Without loss of generality we work with zero interest rates.

We denote by $\mathbb{P}_{a}\left(\mathbb{P}_{e}\right)$ the set of local martingale measures absolutely continuous (equivalent) to $P$. To avoid trivialities we assume $\mathbb{P}_{e} \neq \emptyset$.

In the market we also have $n$ contingent claims (i.e. $\mathcal{F}_{T}$-measurable random variables) $G_{1}, \cdots, G_{n}$. We write $G=\left(G_{1}, \cdots, G_{n}\right)$. Since we are working with exponential utility, we shall assume throughout that $G$ is bounded.

Let us consider the possible no-arbitrage price vectors of $G$.

Definition 2.1 We call $p \in \mathbf{R}^{n}$ an arbitrage price vector of $G$ if there exist

(i) $\lambda \in \mathbf{R}^{n}$ (a static options position); and

(ii) an $\mathbb{F}$-predictable, $S$-integrable $\mathbf{R}^{d}$-valued process $\theta$ (a dynamic trading strategy in the stocks and money-market account) for which $\int_{0}^{t} \theta \cdot d S$ is uniformly bounded below,

such that

$$
\begin{aligned}
& \lambda \cdot(G-p)+\int_{0}^{T} \theta \cdot d S \geq 0 \quad \text { a.s. with } \\
& P\left(\lambda \cdot(G-p)+\int_{0}^{T} \theta \cdot d S>0\right)>0 .
\end{aligned}
$$

We call any $p \in \mathbf{R}^{n}$ that is not an arbitrage price vector, a no-arbitrage price vector.

We define

$$
V=V^{G}:=\left\{E^{Q}[G]=\left(E^{Q}\left[G_{1}\right], \ldots, E^{Q}\left[G_{n}\right]\right) \mid Q \in \mathbb{P}_{e}\right\} \subset \mathbf{R}^{n} .
$$


Proposition 2.1 The set $V$ is convex and coincides with the set of no-arbitrage price vectors for $G$.

Proof That $V$ is convex is obvious as it is the image of the convex set $\mathbb{P}_{e}$ under the linear map $Q \mapsto E^{Q}[G]$. It is therefore determined by its projections $V_{\lambda}:=$ $\{\lambda \cdot p \mid p \in V\}$ for all $\lambda \in \mathbf{R}^{n}$; see Lemma A.1. By [21, Theorem 5.3] applied to the claim $\lambda \cdot G$, there are two cases. Either $V_{\lambda}=\left\{E^{Q}[\lambda \cdot G] \mid Q \in \mathbb{P}_{e}\right\}$ is a point which is the unique no-arbitrage price of $\lambda \cdot G$, or it is an open interval consisting of all no-arbitrage prices of $\lambda \cdot G$.

As follows from this proof, $V$ is either an open subset of $\mathbf{R}^{n}$ or contained in a hyperplane (see Lemma A.1). We shall assume:

Assumption 2.1 $V$ is an open subset of $\mathbf{R}^{n}$.

Remark 2.1 If $V$ were contained in a hyperplane, then $V_{\lambda}$ is a point for some $\lambda$. Then, by [21, Theorem 5.3], the claim $\lambda \cdot G$ can be replicated by a dynamic trading strategy in the stocks and money-market account. This means one or more options is redundant.

Remark 2.2 Under Assumption 2.1, a point $p \in \mathbf{R}^{n}$ belongs to the boundary of the open set $V$ if and only if there exists $\lambda \in \mathbf{R}^{n}$ such that $\lambda \cdot p$ is the superhedging price of the claim $\lambda \cdot G$. Thus $V$ is a natural higher-dimensional analogue of the interval of no-arbitrage prices of a single claim.

\section{Exponential utility}

We shall consider self-financing trading strategies $\theta$ belonging to a suitable space $\Theta$ to be made precise in Definition 4.1 below. A trading strategy $\theta$ with initial capital $x$ leads to terminal wealth $X_{T}^{\theta, x}$, defined from (1.1). Given the derivative security vector $G$ and $\lambda \in \mathbf{R}^{n}$ consider the following value function

$$
u(x ; \lambda):=-\frac{1}{\gamma} \inf _{\theta \in \Theta} \log E\left[\exp \left(-\gamma\left(X_{T}^{\theta, x}+\lambda \cdot G\right)\right)\right] .
$$

The function $u$ measures utility at time $T$ from wealth arising from the option portfolio $\lambda \cdot G$ and the value of a self-financing trading strategy starting with capital $x$. (The log is taken for convenience of presentation and for the convexity statement in Theorem 3.1 below.)

Now suppose that the market price of the security vector $G$ is given by $p=$ $\left(p_{1}, \ldots, p_{n}\right) \in V$. The investor starts with initial wealth $x, \lambda \cdot p$ of which is used to buy the options while the remaining is invested optimally in stock and money market account. Our investor is therefore trying to maximize the quantity

$$
u(x-\lambda \cdot p ; \lambda)
$$

over $\lambda \in \mathbf{R}^{n}$. In order to prove that the maximum is attained, we need the technical assumption that there exists a local martingale measure of finite relative entropy: see (4.1) below. Our main result is then: 
Theorem 3.1 Under assumption (4.1) there exists, for any price vector $p \in V$, a unique static position $\lambda^{*}=\lambda^{*}(p) \in \mathbf{R}^{n}$ (independent of $x$ ) which maximizes $u(x-\lambda \cdot p ; \lambda)$. Moreover, the value function

$$
p \mapsto u\left(x-\lambda^{*}(p) \cdot p ; \lambda^{*}(p)\right)
$$

is strictly convex and continuously differentiable on $V$. Its gradient at $p \in V$ is $\lambda^{*}(p)$.

Remark 3.1 Clearly, for $p \notin V, \sup _{\lambda} u(x-\lambda \cdot p ; \lambda)=+\infty$.

\section{Convex duality}

The main tool for proving Theorem 3.1 is convex duality, in particular the relation between exponential utility and relative entropy. Recall that if $Q$ is any probability measure, then the relative entropy $H(Q \mid P)$ is defined by

$$
H(Q \mid P)= \begin{cases}E\left[\frac{d Q}{d P} \log \frac{d Q}{d P}\right] & \text { if } Q \ll P \\ +\infty & \text { otherwise }\end{cases}
$$

Define $\mathbb{P}_{f}=\mathbb{P}_{f}(P)$ to be the set of $Q \in \mathbb{P}_{a}$ with finite relative entropy, $H(Q \mid P)<\infty$. To proceed we make the assumption

$$
\mathbb{P}_{f} \cap \mathbb{P}_{e} \neq \emptyset \text {. }
$$

There is then a unique measure $Q^{0} \in \mathbb{P}_{f} \cap \mathbb{P}_{e}$ minimizing $H(Q \mid P)$ over all $Q \in \mathbb{P}_{f}$ : see [13, Prop. 3.1].

We can now define the set of allowable strategies.

Definition 4.1 We denote by $\Theta$ the set of $\mathbb{F}$-predictable, $S$-integrable $\mathbf{R}^{d}$-valued processes $\theta$ such that the wealth processes $X^{\theta, 0}$, defined by (1.1), are $Q$ martingales for all $Q \in \mathbb{P}_{f}$.

We then have

Theorem 4.1 ( [6, Theorem 2.2] and [13, Theorem 2.1])

$$
u(x ; \lambda)=x+\inf _{Q \in \mathbb{P}_{f}}\left\{\lambda \cdot E^{Q}[G]+\frac{1}{\gamma} H(Q \mid P)\right\} .
$$

Moreover, the infimum is attained for a unique $Q^{\lambda} \in \mathbb{P}_{f} \cap \mathbb{P}_{e}$ whose Radon-Nikodym derivative is given by

$$
\frac{d Q^{\lambda}}{d P}=\exp \left(c_{\lambda}-\gamma\left(\int_{0}^{T} \theta^{\lambda} \cdot d S+\lambda \cdot G\right)\right)
$$

where $\theta^{\lambda} \in \Theta$ attains the infimum in the primal problem (3.1), and $c_{\lambda}$ is a constant. 


\section{Utility indifference pricing}

Consider an investor who at time zero is offered to buy an option, or a set of options, with payoff at time $T$. The price for which this investor is indifferent to the investment opportunity, in terms of maximum expected utility, is called the buyer's indifference price of the option. The definition of indifference prices asks the question at what price $b(\lambda)$ is the buyer indifferent between holding the options portfolio $\lambda \cdot G$ or no options at all, while we are interested in how many of each type of option to optimally invest in, given the market price $p$. We make the connection between the two problems in Section 7.

Definition 5.1 The indifference price $b(\lambda)$ of the options portfolio $\lambda \cdot G$ is defined by the following equation:

$$
u(x-b(\lambda) ; \lambda)=u(x ; 0),
$$

with $u$ given in (4.2).

Remark 5.1 Strictly speaking we should have denoted the buyer's price by $b(\lambda ; x)$, but (4.2) shows that it does not depend on the buyer's wealth $x$. This is an artifact of exponential utility and will not hold in more general situations. An alternative definition of the indifference price $b(\lambda)$ is $u(x ; \lambda)=u(x+b(\lambda) ; 0)$. For exponential utility, this is equivalent to (5.1), whereas it may differ in the case of other utility functions.

In view of (4.2), (5.1) leads to

$$
\begin{aligned}
b(\lambda) & =\inf _{Q \in \mathbb{P}_{f}}\left\{\lambda \cdot E^{Q}[G]+\frac{1}{\gamma} H(Q \mid P)\right\}-\inf _{Q \in \mathbb{P}_{f}} \frac{1}{\gamma} H(Q \mid P) \\
& =\inf _{Q \in \mathbb{P}_{f}}\left\{\lambda \cdot E^{Q}[G]+\frac{1}{\gamma}\left(H(Q \mid P)-H\left(Q^{0} \mid P\right)\right)\right\},
\end{aligned}
$$

where $Q^{0} \in \mathbb{P}_{f} \cap \mathbb{P}_{e}$ minimizes $H(Q \mid P)$. We shall prove

Theorem 5.1 The function $\lambda \mapsto b(\lambda)$ is strictly concave and continuously differentiable on $\mathbf{R}^{n}$. Its gradient is given by

$$
\nabla b(\lambda)=E^{Q^{\lambda}}[G]
$$

where $Q^{\lambda}$ is defined by (4.3).

\section{Proof of Theorem 5.1}

Notice that for fixed $Q \in \mathbb{P}_{f} \cap \mathbb{P}_{e}$, the function

$$
\lambda \mapsto \lambda \cdot E^{Q}[G]+\frac{1}{\gamma}\left(H(Q \mid P)-H\left(Q^{0} \mid P\right)\right)
$$

is affine. Thus (5.2) directly shows that $b$ is an infimum of affine functions on $\mathbf{R}^{n}$, hence is concave. 
Differentiability and strict concavity are trickier to establish. For $\lambda \in \mathbf{R}^{n}$, define a new probability measure $P^{\lambda} \sim P$ by

$$
\frac{d P^{\lambda}}{d P}=\exp \left(a_{\lambda}-\gamma \lambda \cdot G\right)
$$

for a suitable constant $a_{\lambda} \in \mathbf{R}$. A direct computation shows that

$$
H\left(Q \mid P^{\lambda}\right)=H(Q \mid P)+\gamma \lambda \cdot E^{Q}[G]-a_{\lambda}
$$

for any $Q \in \mathbb{P}_{a}$ (recall that $G$ is bounded). It follows that $\mathbb{P}_{f}\left(P^{\lambda}\right)=\mathbb{P}_{f}(P)$. Using (6.1) in (5.2), we obtain

$$
b(\lambda)=\inf _{Q \in \mathbb{P}_{f}} \frac{1}{\gamma} H\left(Q \mid P^{\lambda}\right)-\frac{1}{\gamma} H\left(Q^{0} \mid P\right)+a_{\lambda} .
$$

Now pick $\lambda \in \mathbf{R}^{n}, \mu \in \mathbf{R}^{n}$ and $\epsilon>0$. From (6.1),

$$
b(\lambda+\epsilon \mu)=\inf _{Q \in \mathbb{P}_{f}}\left\{\epsilon \mu \cdot E^{Q}[G]+\frac{1}{\gamma} H\left(Q \mid P^{\lambda}\right)\right\}-\frac{1}{\gamma} H\left(Q^{0} \mid P\right)+a_{\lambda} .
$$

Therefore,

$$
\begin{aligned}
\frac{b(\lambda+\epsilon \mu)-b(\lambda)}{\epsilon} & =\inf _{Q \in \mathbb{P}_{f}}\left\{\mu \cdot E^{Q}[G]+\frac{1}{\epsilon \gamma} H\left(Q \mid P^{\lambda}\right)\right\}-\inf _{Q \in \mathbb{P}_{f}} \frac{1}{\epsilon \gamma} H\left(Q \mid P^{\lambda}\right) \\
& =\inf _{Q \in \mathbb{P}_{f}}\left\{\mu \cdot E^{Q}[G]+\frac{1}{\epsilon \gamma}\left(H\left(Q \mid P^{\lambda}\right)-H\left(Q^{\lambda} \mid P^{\lambda}\right)\right)\right\},
\end{aligned}
$$

where $Q^{\lambda}$ is the unique minimizer of $H\left(Q \mid P^{\lambda}\right)$. It is given by (4.3), as follows from Theorem 4.1.

Comparing with (5.2) shows that expression (6.2) is the indifference price of the claim $\mu \cdot G$ for a buyer with subjective measure $P^{\lambda}$ and risk-aversion parameter $\epsilon \gamma$. Letting $\epsilon \downarrow 0$ corresponds to the limit of zero risk-aversion which was shown by Becherer [3, Proposition 3.2] (see also [18, Theorem 5.2]) to be the expected payoff under the minimal entropy martingale measure. Hence $b$ admits a gradient at $\lambda$ and $\nabla b(\lambda)=E^{Q^{\lambda}}[G]$. Since this is true for all $\lambda$ and $b$ is concave, it follows that $b$ is in fact continuously differentiable: see Lemma A.2.

Now pick $\lambda_{1}, \lambda_{2} \in \mathbf{R}^{n}$ such that $\lambda_{1} \neq \lambda_{2}$. Let us assume that $b$ is affine on the line segment between $\lambda_{1}$ and $\lambda_{2}$ and derive a contradiction. This will show that $b$ is strictly concave and complete the proof.

Write $P^{i}=P^{\lambda_{i}}$ and $Q^{i}=Q^{\lambda_{i}}$ for $i=1,2$. From (6.1), we obtain

$$
\begin{aligned}
& \left(H\left(Q^{2} \mid P^{1}\right)-H\left(Q^{1} \mid P^{1}\right)\right)+\left(H\left(Q^{1} \mid P^{2}\right)-H\left(Q^{2} \mid P^{2}\right)\right) \\
& =\gamma\left(\lambda_{1}-\lambda_{2}\right) \cdot\left(E^{Q^{2}}[G]-E^{Q^{1}}[G]\right)=0,
\end{aligned}
$$

where the last equality follows from the assumption that $b$ is affine on the line segment and the previous computation of the gradient of $b$. The left hand side is a sum of two nonnegative terms, hence both are zero. As $Q^{i}$ is the unique minimizer of $H\left(Q \mid P^{i}\right)$ this implies $Q^{1}=Q^{2}$. 
Applying (4.3) with $\lambda=\lambda_{1}, \lambda_{2}$ and using $Q^{1}=Q^{2}$ we find

$$
\nu \cdot G=c+\int_{0}^{T} \theta \cdot d S
$$

for some $\theta \in \Theta$, and where $\nu=\lambda_{2}-\lambda_{1}$ and $c$ is a constant. By the definition of $\Theta$, $E^{Q}[\nu \cdot G]=c$ for all $Q \in \mathbb{P}_{f} \cap \mathbb{P}_{e}$. Lemma 3.4 in [6] shows that $\left\{\frac{d Q}{d P} \mid Q \in \mathbb{P}_{f} \cap \mathbb{P}_{e}\right\}$ is $L^{1}(P)$-dense in $\left\{\frac{d Q}{d P} \mid Q \in \mathbb{P}_{e}\right\}$. Therefore, $E^{Q}[\nu \cdot G]=c$ for all $Q \in \mathbb{P}_{e}$. Hence $V_{\nu}=\{c\}$, contradicting Assumption 2.1 and completing the proof of Theorem 5.1.

\section{Proof of Theorem 3.1}

For fixed $p \in V$ we have to maximize $u(x-\lambda \cdot p ; \lambda)$ over $\lambda \in \mathbf{R}^{n}$. But comparing (4.2) with (5.2) we see that

$$
u(x-\lambda \cdot p ; \lambda)=b(\lambda)-\lambda \cdot p+x+u(0 ; 0) .
$$

Thus we have to maximize $b(\lambda)-\lambda \cdot p$.

While the set $V$ of no-arbitrage price vectors was defined in terms of all equivalent local martingale measures, the following lemma shows that it suffices to consider measures of the form $Q^{\lambda}$.

Lemma 7.1 $V=\left\{\nabla b(\lambda) \mid \lambda \in \mathbf{R}^{n}\right\}=\left\{E^{Q^{\lambda}}[G] \mid \lambda \in \mathbf{R}^{n}\right\}$.

From this lemma and from the strict concavity and continuous differentiability of $b$, it follows that there exists a unique $\lambda^{*}=\lambda^{*}(p)$ for which $\nabla b\left(\lambda^{*}\right)=p$, and this $\lambda^{*}$ is the unique maximizer of $b(\lambda)-\lambda \cdot p$. The function $p \mapsto u\left(x-\lambda^{*}(p) \cdot p ; \lambda^{*}(p)\right)$ is then, up to an additive constant, the Fenchel-Legendre transform of $b$ and hence a strictly convex function on $V$ with gradient $\lambda^{*}(p)$.

Let us complete the proof of Theorem 3.1 by proving Lemma 7.1 .

Proof of Lemma 7.1 The second equality is a consequence of (5.2). As $b$ is strictly concave with a well-defined gradient everywhere it follows that the set $V^{\prime}:=$ $\left\{\nabla b(\lambda) \mid \lambda \in \mathbf{R}^{n}\right\}$ is open and convex, hence determined by its projections $V_{\lambda}^{\prime}:=$ $\lambda \cdot V^{\prime}$. See Lemma A.3 and Lemma A.1. It is clear that $V^{\prime} \subset V$, and it suffices to show $V_{\lambda} \subset V_{\lambda}^{\prime}$ for every $\lambda \in \mathbf{R}^{n}$.

Let $\phi(t):=b(t \lambda)$ be the buyer's indifference price of the claim $t \lambda \cdot G$. Then $\phi(t)$ is differentiable and strictly concave on $\mathbf{R}$, and it follows from [3, Corollary 3.3] that

$$
\lim _{t \rightarrow \infty} \phi^{\prime}(t)=\lim _{t \rightarrow \infty} \frac{\phi(t)}{t}=\inf _{Q \in \mathbb{P}_{e}} E^{Q}[\lambda \cdot G] \quad \text { and } \quad \lim _{t \rightarrow-\infty} \phi^{\prime}(t)=\sup _{Q \in \mathbb{P}_{e}} E^{Q}[\lambda \cdot G] .
$$

See Lemma A.4 for the first equality above. Thus $V_{\lambda}=\left\{\phi^{\prime}(t) \mid t \in \mathbf{R}\right\}$. But, $\phi^{\prime}(t)=\lambda \cdot \nabla b(t \lambda) \in V_{\lambda}^{\prime}$, so $V_{\lambda} \subset V_{\lambda}^{\prime}$, which completes the proof. 


\section{Concluding remarks}

We have shown that the optimal investment problem with derivative securities has a unique solution under natural arbitrage-free conditions on the market prices of the derivatives in the case of bounded payoffs and exponential utility. In particular, the continuous differentiability and the strict concavity of the indifference price have been key to establishing this result. It is natural to ask about other utility functions for which there exist quite general duality results, albeit not as explicit as the duality formula (4.2). The main results for general utility functions defined on $\mathbf{R}_{+}$can be found in [14] when there is no claim, and in [4] when there is a claim. For general utility functions defined on $\mathbf{R}$, the case without a claim is studied in [20] and with a claim in [16]. Since our analysis utilizes the simple conjugation between the primal and dual value functions and the zero risk aversion limit, extension to more general cases is not trivial. Some recent results in this direction appear in $[10,11]$.

\section{Appendix: Results from convex analysis}

Here we gather some results from convex analysis that we need in the paper.

Lemma A.1 For $\emptyset \neq V \subset \mathbf{R}^{n}$ and $\lambda \in \mathbf{R}^{n}$ write $V_{\lambda}:=\{\lambda \cdot p \mid p \in V\} \subset \mathbf{R}$. Then:

(i) $V$ is contained in a hyperplane if and only if $V_{\lambda}$ is a point for some $\lambda$;

(ii) if $V$ is convex, then $V_{\lambda}$ is a point or an interval for all $\lambda$;

(iii) if $V$ is convex, then $V$ is open if and only if $V_{\lambda}$ is open for all $\lambda$;

(iv) if $V$ and $V^{\prime}$ are open and convex, then $V=V^{\prime}$ if and only if $V_{\lambda}=V_{\lambda}^{\prime}$ for all $\lambda$.

Proof The first assertion is trivial. The second holds since $V$ is connected and $p \mapsto \lambda \cdot p$ is continuous, hence $V_{\lambda}$ is connected. As for (iii), if $V$ is open, then the affine function $p \mapsto \lambda \cdot p$ cannot attain a local maximum or minimum on $V$, hence $V_{\lambda}$ is open. On the other hand, if $V$ is convex but not open and $p \in V \cap \partial V$, then there exists a hyperplane through $p$ and disjoint from int $(V)$. Thus there exists $\lambda$ such that $\lambda \cdot p \geq \lambda \cdot q$ for all $q \in V$ and $V_{\lambda}$ is not open. Finally, to prove (iv), assume $V \not \subset V^{\prime}$ and pick $p \in V \backslash V^{\prime}$. As $V^{\prime}$ is open, there exists a hyperplane through $p$, separating $p$ from $V^{\prime}$, i.e. there exists $\lambda$ such that $\lambda \cdot p>\lambda \cdot p^{\prime}$ for all $p^{\prime} \in V^{\prime}$. Thus $V_{\lambda} \neq V_{\lambda}^{\prime}$

Lemma A.2 If $\phi: \mathbf{R}^{n} \rightarrow \mathbf{R}$ is concave and admits a gradient $\nabla \phi(x) \in \mathbf{R}^{n}$ for all $x \in \mathbf{R}^{n}$, in the sense that $\lim _{t \rightarrow 0}(\phi(x+t y)-\phi(x)) / t=\nabla \phi(x) \cdot y$ for every $y \in \mathbf{R}^{n}$, then $\phi$ is continuously differentiable in the sense that $x \mapsto \nabla \phi(x)$ is continuous.

Proof See [7, p.86] or [17, Theorem 25.5].

Lemma A.3 If $\phi: \mathbf{R}^{n} \rightarrow \mathbf{R}$ is continuously differentiable and strictly concave, then the set $V:=\left\{\nabla \phi(x) \mid x \in \mathbf{R}^{n}\right\}$ is open and convex. 
Proof The set $V$ is the interior of the locus where the (convex) Fenchel-Legendre transform of $\phi$ is finite-valued, and is hence convex. See [7, p.104] or [17, Theorem 26.5].

Lemma A.4 If $\phi: \mathbf{R} \rightarrow \mathbf{R}$ is continuously differentiable and concave, then the limit $\phi^{\prime}(\infty):=\lim _{t \rightarrow \infty} \phi^{\prime}(t)$ exists (it may be $-\infty$ ). Moreover, $\phi^{\prime}(\infty)=$ $\lim _{t \rightarrow \infty} \phi(t) / t$.

Proof The limit defining $\phi^{\prime}(\infty)$ exists as $\phi^{\prime}$ is decreasing. Assume $\phi^{\prime}(\infty) \neq-\infty$ for simplicity. Fix $\epsilon>0$ and pick $t_{0}$ large enough so that $\phi^{\prime}\left(t_{0}\right)<\phi^{\prime}(\infty)+\epsilon$. By concavity,

$$
\frac{\phi(t)-\phi\left(t_{0}\right)}{t-t_{0}}-\epsilon \leq \phi^{\prime}\left(t_{0}\right)-\epsilon \leq \phi^{\prime}(\infty) \leq \frac{\phi(t)-\phi\left(t_{0}\right)}{t-t_{0}}
$$

for $t \geq t_{0}$. Letting $t \rightarrow \infty$ yields $\lim \sup \phi(t) / t-\epsilon \leq \phi^{\prime}(\infty) \leq \lim \inf \phi(t) / t$. As $\epsilon \rightarrow 0$ we get $\lim \phi(t) / t=\phi^{\prime}(\infty)$. The case when $\phi^{\prime}(\infty)=-\infty$ is easier and we omit the details.

\section{References}

1. Barrieu, P., El Karoui, N.: Optimal design of derivatives in illiquid markets. Quant. Finance 2, 181-188 (2002)

2. Barrieu, P., El Karoui, N.: Inf-convolution of risk measures and optimal risk transfer. Finance Stochast. 9, 269-298 (2005)

3. Becherer, D.: Rational hedging and valuation of integrated risks under constant absolute risk aversion. Insurance Math. Econ. 33, 1-28 (2003)

4. Cvitanić, J., Schachermayer, W., Wang, H.: Utility maximization in incomplete markets with random endowment. Finance Stochast. 5, 259-272 (2001)

5. Davis, M.: Option pricing in incomplete markets. In: Dempster, M.A.H., Pliska, S.R. (eds.) Mathematics of derivative securities. Cambridge: Cambridge University Press 1998, pp. 216-226

6. Delbaen, F., Grandits, P., Rheinländer, T., Samperi, D., Schweizer, M., Stricker, C.: Exponential hedging and entropic penalties. Math. Finance 12, 99-123 (2002)

7. Fenchel, W.: Convex cones, sets and functions (Lecture Notes). Princeton: Princeton University 1951

8. Frittelli, M.: The minimal entropy martingale measure and the valuation problem in incomplete markets. Math. Finance 10, 39-52 (2000)

9. Hodges, S.D., Neuberger, A.: Optimal replication of contingent claims under transaction costs. Rev. Futures Markets 8, 222-239 (1989)

10. Hugonnier, J., Kramkov, D.: Optimal investment with random endowment in incomplete markets. Ann. Appl. Probab. 14, 845-864 (2004)

11. Hugonnier, J., Kramkov, D., Schachermayer, W.: On utility based pricing of contingent claims in incomplete markets. Math. Finance, (2004)

12. İlhan, A., Jonsson, M., Sircar, S.: Portfolio optimization with derivatives and indifference pricing. In: Carmona, R. (ed.) Volume on indifference pricing. Princeton: Princeton University Press 2005

13. Kabanov, Y. M., Stricker, C.: On the optimal portfolio for the exponential utility maximization: Remarks to the six-author paper. Math. Finance 12, 125-134 (2002)

14. Kramkov, D., Schachermayer, W.: The asymptotic elasticity of utility functions and optimal investment in incomplete markets. Ann. Appl. Probab. 9, 904-950 (1999)

15. Musiela, M., Zariphopoulou, T.: An example of indifference prices under exponential preferences. Finance Stochast. 8, 229-239 (2004) 
16. Owen, M. P.: Utility based optimal hedging in incomplete markets. Ann. Appl. Probab. 12, 691-709 (2002)

17. Rockafellar, R. T.: Convex analysis. Princeton: Princeton University Press 1970

18. Rouge, R., El Karoui, N.: Pricing via utility maximization and entropy. Math. Finance 10, 259-76 (2000)

19. Schachermayer, W.: Optimal investment in incomplete financial markets. In: Geman, H. et al.(eds.) Mathematical finance: Bachelier Congress 2000. Berlin Heidelberg New York: Springer 2001, pp. $427-462$

20. Schachermayer, W.: Optimal investment in incomplete markets when wealth may become negative. Ann. Appl. Probab. 11, 694-734 (2001)

21. Schachermayer, W.: Introduction to the mathematics of financial markets. In: Bernard, P. (ed.) Lectures on probability theory and statistics, Saint-Flour summer school 2000. (Lecture Notes in Mathematics 1816). Berlin Heidelberg New York: Springer 2003, pp. 111-177

22. Sircar, R., Zariphopoulou, T.: Bounds and asymptotic approximations for utility prices when volatility is random. SIAM J. Control Opt. 43, 1328-1353 (2005) 